\title{
Tetrahedral and Pentatopic Sum Labeling of Graphs
}

\author{
S. Murugesan \\ Department of Mathematics \\ C. B. M. College, Kovaipudur, \\ Coimbatore - 641042 , \\ Tamil Nadu, INDIA.
}

\author{
D. Jayaraman \\ Department of Mathematics \\ C. B. M. College, Kovaipudur, \\ Coimbatore - 641 042, Tamil Nadu, INDIA.
}

\author{
J. Shiama \\ Department of Mathematics, \\ C. M. S. College of Science and Commerce, \\ Coimbatore- 641 049, Tamil Nadu, INDIA.
}

\begin{abstract}
A $(p, q)$ graph $G$ is said to admit tetrahedral or pentatopic sum labeling if its vertices can be labeled by non negative integers such that the induced edge labels obtained by the sum of the labels of end vertices are the first q tetrahedral or pentatope numbers. A graph $G$ which admits tetrahedral or pentatopic sum labeling is called tetrahedral or pentatopic sum graph. In this paper we prove that paths, combs, stars, subdivision of stars and $B_{m, n}$ admit tetrahedral and pentatopic sum labeling.
\end{abstract}

\section{Keywords:}

Tetrahedral sum labeling, pentatopic sum labeling.

\section{INTRODUCTION}

The graphs considered here are finite, connected, undirected and simple. The vertex set and edge set of a graph $G$ are denoted by $V(G)$ and $E(G)$ respectively. For various graph theoretic notations and terminology we follow Harary [1] and for number theory we follow Burton [2]. We will give the brief summary of definitions which are useful for the present investigations.

DEFINITION 1. If the vertices of the graph are assigned values subject to certain conditions it is known as graph labeling.

A dynamic survey on graph labeling is regularly updated by Gallian [3] and it is published by Electronic Journal of Combinatorics. Vast amount of literature is available on different types of graph labeling and more than 1000 research papers have been published so far in last four decades.

Most important labeling problems have three important ingredients.

* A set of numbers from which vertex labels are chosen;

* A rule that assigns a value to each edge;

* A condition that these values must satisfy.

The present work is to aimed to discuss two such labelings known as tetrahedral sum labeling and pentatopic sum labeling.

DEFINITION 2. A triangular number is a number obtained by adding all positive integers less than or equal to a given positive integer $n$. If the $n^{\text {th }}$ triangular number is denoted by $A_{n}$, then $A_{n}=\frac{1}{2} n(n+1)$.

The triangular numbers are $1,3,6,10,15,21,28,36,45,55, \ldots$

DEFINITION 3. A triangular sum labeling of a graph $G$ is a one-to-one function $f: V(G) \rightarrow N$ (where $N$ is the set of all non-negative integers) that induces a bijection $f^{+}: E(G) \rightarrow$ $\left\{A_{1}, A_{2}, \ldots, A_{q}\right\}$ of the edges of $G$ defined by $f^{+}(u v)=f(u)+$ $f(v), \forall e=u v \in E(G)$. The graph which admits such labeling is called a triangular sum graph.

This concept was introduced by Hegde and Shankaran [4]. Motivated by the concept of triangular sum labeling, we introduce two new types of labeling called tetrahedral sum labeling and pentatopic sum labeling.
DEFINITION 4. A tetrahedral number (or triangular pyramidal number) is a figurative number that represents a pyramid with a triangular base and three sides, called a tetrahedron. The $n^{\text {th }}$ tetrahedral number is the sum of the first $n$ triangular numbers. If the $n^{\text {th }}$ tetrahedral number is denoted by $B_{n}$, then $B_{n}=\frac{1}{6} n(n+1)(n+2)$. The tetrahedral numbers are $1,4,10,20,35,56,84,120,165,220,286,364,455,560,680$, $816,969, \ldots$

DEFINITION 5. A tetrahedral sum labeling of a graph $G$ is a one-to-one function $f: V(G) \rightarrow N$ that induces a bijection $f^{+}: E(G) \rightarrow\left\{B_{1}, B_{2}, \ldots, B_{q}\right\}$ of the edges of $G$ defined by $f^{+}(u v)=f(u)+f(v), \forall e=u v \in E(G)$. The graph which admits such labeling is called a tetrahedral sum graph.

EXAMPLE 1. A tetrahedral sum graph with 8 vertices is shown below.

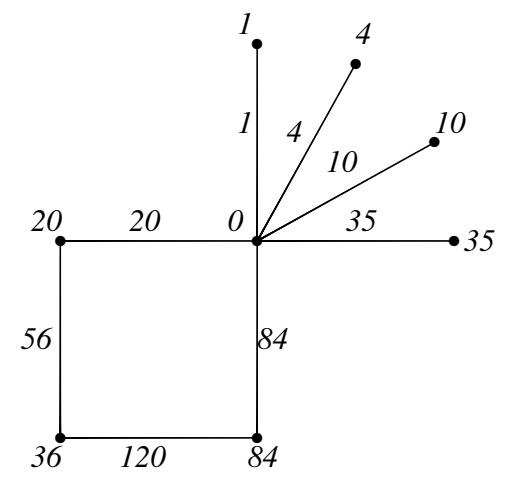

DEFINITION 6. A pentatope number is a figurative number given by Ptop $=\frac{1}{4} B_{n}(n+3)$ where $B_{n}$ is the $n^{\text {th }}$ tetrahedral number. The $n^{\text {th }}$ pentatope number is the sum of the first $n$ tetrahedral numbers. If the $n^{\text {th }}$ pentatope number is denoted by $C_{n}$, then $C_{n}=$ $\frac{1}{24} n(n+1)(n+2)(n+3)$. The pentatopic numbers are $1,5,15,35,70,126,210,330,495,715,1001,1365,1820,2380,3060$, $3876,4845, \ldots$

DEFINITION 7. A pentatopic sum labeling of a graph $G$ is a one-to-one function $f: V(G) \rightarrow N$ that induces a bijection $f^{+}: E(G) \rightarrow\left\{C_{1}, C_{2}, \ldots, C_{q}\right\}$ of the edges of $G$ defined by $f^{+}(u v)=f(u)+f(v), \forall e=u v \in E(G)$. The graph which admits such labeling is called a pentatopic sum graph.

EXAMPLE 2. A pentatopic sum graph with 13 vertices is shown below. 


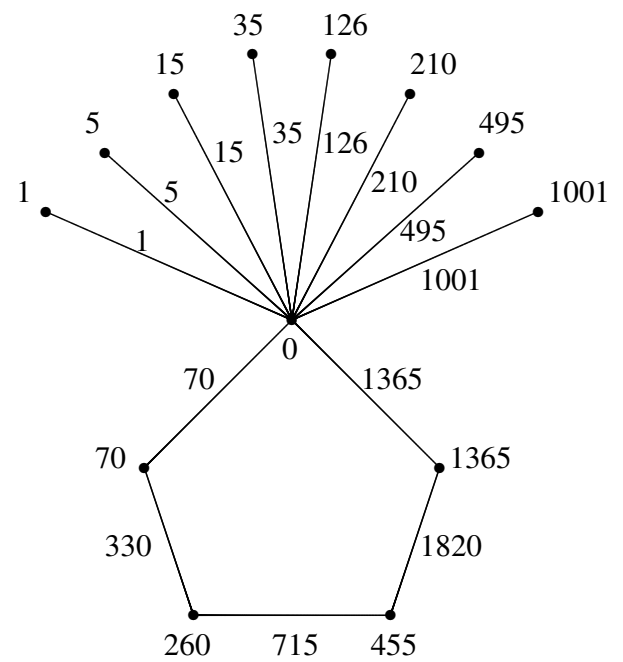

\section{MAIN RESULTS}

Here we prove that paths, combs, stars, double stars and bistars admit tetradedral and pentatopic sum labeling.

\section{THEOREM 1. The path $P_{n}$ admits tetrahedral sum labeling.}

Proof. Let $P_{n}: u_{1} u_{2} \ldots u_{n}$ be the path and let $v_{i}=$ $u_{i} u_{i+1}(1 \leq \mathrm{i} \leq \mathrm{n}-1)$ be the edges.

For $i=1,2, \ldots, n$, define

$$
f\left(u_{i}\right)= \begin{cases}\frac{1}{24}(i-1)(i+1)(2 i+3) & \text { if } \mathrm{i} \text { is odd } \\ \frac{1}{24} i(i+2)(2 i-1) & \text { if } \mathrm{i} \text { is even }\end{cases}
$$

We will prove that the induced edge labels obtained by the sum of the labels of end vertices are the first $n-1$ tetrahedral numbers.

Case(i): $i$ is odd

For $1 \leq i \leq n-1$,

$$
\begin{aligned}
f\left(u_{i}\right)+f\left(u_{i+1}\right)= & \frac{1}{24}(i-1)(i+1)(2 i+3) \\
& +\frac{1}{24}(i+1)(i+3)(2 i+1) \\
= & \frac{1}{24}(i+1)[(i-1)(2 i+3) \\
& +(i+3)(2 i+1)] \\
= & \frac{1}{6} i(i+1)(i+2) \\
= & B_{i} \\
= & f^{+}\left(v_{i}\right.
\end{aligned}
$$

Case(ii): $\mathrm{i}$ is even

For $1 \leq i \leq n-1$,

$$
\begin{aligned}
f\left(u_{i}\right)+f\left(u_{i+1}\right) & =\frac{1}{24} i(i+2)(2 i-1)+\frac{1}{24} i(i+2)(2 i+5) \\
& =\frac{1}{6} i(i+1)(i+2) \\
& =B_{i} \\
& =f^{+}\left(v_{i}\right)
\end{aligned}
$$

Thus the induced edge labels are the first $n-1$ tetrahedral numbers.

Hence path $P_{n}$ admits tetrahedral sum labeling.

EXAMPLE 3. The tetrahedral sum labeling of $P_{11}$ is shown below.

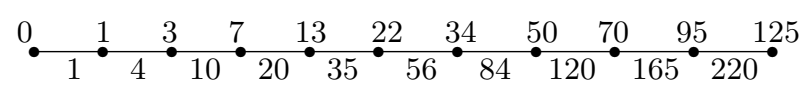

THEOREM 2. The comb $P_{n} \odot K_{1}$ admits tetrahedral sum labeling.

Proof. Let $P_{n}: u_{1} u_{2} \ldots u_{n}$ be the path and let $v_{i}=$ $u_{i} u_{i+1}(1 \leq \mathrm{i} \leq \mathrm{n}-1)$ be the edges. Let $w_{1}, w_{2}, \ldots, w_{n}$ be the pendant vertices adjacent to $u_{1}, u_{2}, \ldots, u_{n}$ respectively and $t_{i}=$ $u_{i} w_{i}(1 \leq \mathrm{i} \leq \mathrm{n}-1)$ be the edges.

For $i=1,2, \ldots, n$, define

$$
f\left(u_{i}\right)= \begin{cases}\frac{1}{24}(i-1)(i+1)(2 i+3) & \text { if } \mathrm{i} \text { is odd } \\ \frac{1}{24} i(i+2)(2 i-1) & \text { if } \mathrm{i} \text { is even }\end{cases}
$$

and

$f\left(w_{i}\right)= \begin{cases}\frac{1}{24}\left[2 i^{3}+(12 n-3) i^{2}\right. & \\ \left.+\left(12 n^{2}-2\right) i+\left(4 n^{3}-4 n+3\right)\right], & \text { if } \mathrm{i} \text { is odd } \\ \frac{1}{24}\left[2 i^{3}+(12 n-3) i^{2}\right. & \\ \left.+\left(12 n^{2}-2\right) i+4 n\left(n^{2}-1\right)\right], & \text { if } \mathrm{i} \text { is even. }\end{cases}$

Then $f\left(u_{i}\right)+f\left(u_{i+1}\right)=f^{+}\left(v_{i}\right)$ for $1 \leq i \leq n-1$

and $f\left(u_{i}\right)+f\left(w_{i}\right)=f^{+}\left(t_{i}\right)$ for $1 \leq i \leq n$.

Thus the induced edge labels are the first $2 n-1$ tetrahedral numbers.

Hence comb admits tetrahedral sum labeling.

EXAMPLE 4. The tetrahedral sum labeling of $P_{6} \odot K_{1}$ is shown below.

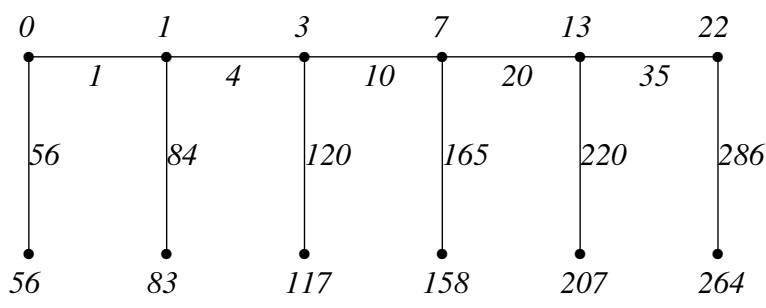


THEOREM 3. The star graph $K_{1, n}$ admits tetrahedral sum labeling.

Proof. Let $v$ be the apex vertex and let $v_{1}, v_{2}, \ldots, v_{n}$ be the pendant vertices of the star $K_{1, n}$.

Define

$$
\begin{aligned}
f(v) & =0 \\
\text { and } f\left(v_{i}\right) & =\frac{1}{6} i(i+1)(i+2), 1 \leq i \leq n .
\end{aligned}
$$

We see that the induced edge labels are the first $n$ tetrahedral numbers.

Hence $K_{1, n}$ admits tetrahedral sum labeling.

EXAMPLE 5. The tetrahedral sum labeling of $K_{1,8}$ is shown below.

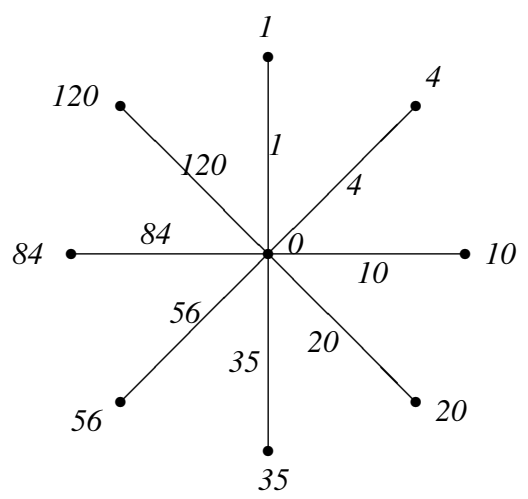

THEOREM 4. $S\left(K_{1, n}\right)$, the subdivision of the star $K_{1, n}$, ad mits tetrahedral sum labeling.

Proof. Let $V\left(S\left(K_{1, n}\right)\right)=\left\{v, v_{i}, u_{i}: 1 \leq i \leq n\right\}$ and $E\left(S\left(K_{1, n}\right)\right)=\left\{v v_{i}, v_{i} u_{i}: 1 \leq i \leq n\right\}$.

Define $f$ by

$$
\begin{aligned}
f(v) & =0 \\
f\left(v_{i}\right) & =\frac{1}{6} i(i+1)(i+2), 1 \leq i \leq n \\
\text { and } f\left(u_{i}\right) & =\frac{1}{6}\left[n^{3}+3 n^{2}+2 n+3 n i(i+n+2)\right], 1 \leq i \leq n .
\end{aligned}
$$

We see that the induced edge labels are the first $2 n$ tetrahedral numbers.

Hence $S\left(K_{1, n}\right)$ admits tetrahedral sum labeling.

EXAMPLE 6. The tetrahedral sum labeling of $S\left(K_{1,6}\right)$ is shown below.

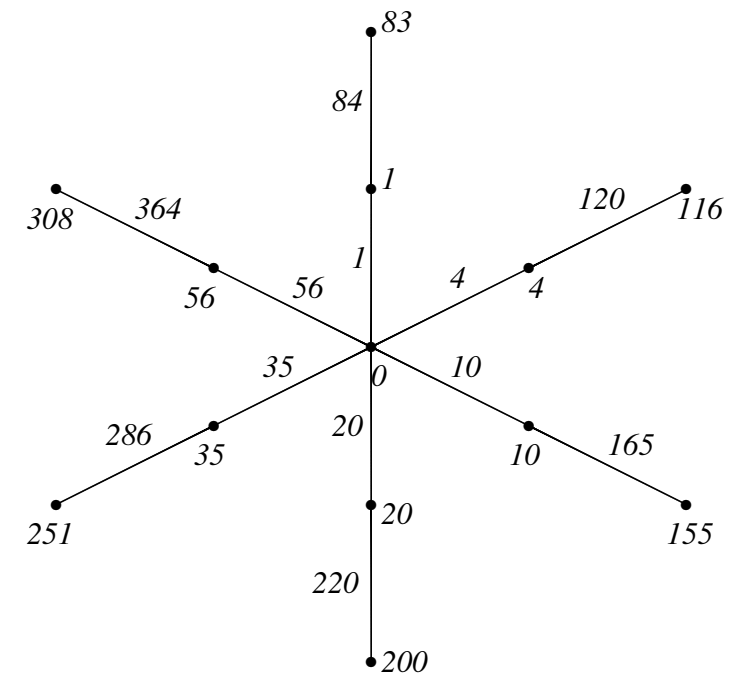

THEOREM 5. The bistar $B_{m, n}$ admits tetrahedral sum labeling.

Proof. Let $V\left(B_{m, n}\right)=\left\{u, v, u_{i}, v_{j}: 1 \leq \mathrm{i} \leq \mathrm{m}, 1 \leq \mathrm{j} \leq \mathrm{n}\right\}$ and

$E\left(B_{m, n}\right)=\left\{u v, u u_{i}, v v_{j}: 1 \leq \mathrm{i} \leq \mathrm{m}, 1 \leq \mathrm{j} \leq \mathrm{n}\right\}$.

Define $f$ by

$$
\begin{aligned}
f(u) & =0, \\
f(v) & =1, \\
f\left(u_{i}\right) & =\frac{1}{6}(i+1)(i+2)(i+3), 1 \leq i \leq m \\
\text { and } f\left(v_{j}\right) & =\frac{1}{6}(m+j+1)(m+j+2)(m+j+3)-1,1 \leq j \leq n .
\end{aligned}
$$

We see that the induced edge labels are the first $m+n+1$ tetrahedral numbers.

Hence $B_{m, n}$ admits tetrahedral sum labeling.

EXAMPLE 7. The tetrahedral sum labeling of $B_{6,4}$ is shown below.

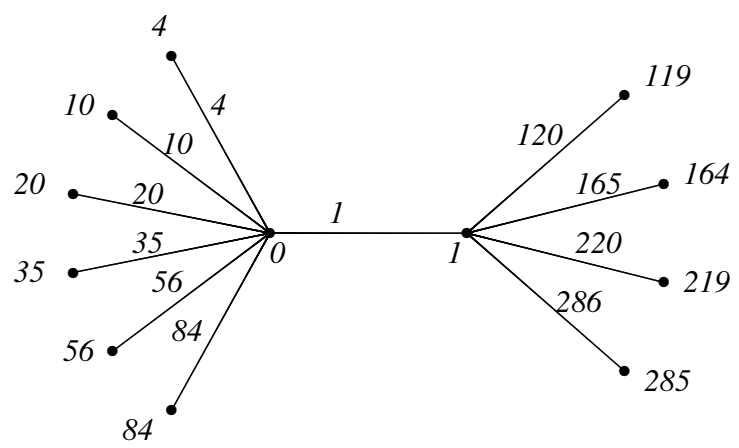

THEOREM 6. The path $P_{n}$ admits pentatopic sum labeling.

PROOF. Let $P_{n}: u_{1} u_{2} \ldots u_{n}$ be the path and let $v_{i}=$ $u_{i} u_{i+1}(1 \leq \mathrm{i} \leq \mathrm{n}-1)$ be the edges. For $i=1,2, \ldots, n$, define 


$$
f\left(u_{i}\right)= \begin{cases}\frac{1}{48}(i-1)\left(i^{3}+5 i^{2}+7 i+3\right) & \text { if } i \text { is odd } \\ \frac{1}{48} i\left(i^{3}+4 i^{2}+2 i-4\right) & \text { if } i \text { is even }\end{cases}
$$

We will prove that the induced edge labels obtained by the sum of the labels of end vertices are the first $n-1$ pentatope numbers.

Case (i): $\mathrm{i}$ is odd

For $1 \leq i \leq n-1$,

$$
\begin{aligned}
f\left(u_{i}\right)+f\left(u_{i+1}\right)= & \frac{1}{48}(i-1)\left(i^{3}+5 i^{2}+7 i+3\right) \\
& +\frac{1}{48}(i+1)\left[(i+1)^{3}+4(i+1)^{2}\right. \\
& +2(i+1)-4] \\
= & \frac{1}{48}(i-1)\left(i^{3}+5 i^{2}+7 i+3\right) \\
& +\frac{1}{48}(i+1)\left(i^{3}+7 i^{2}+13 i+3\right) \\
= & \frac{1}{24} i\left(i^{3}+6 i^{2}+11 i+6\right) \\
= & \frac{1}{24} i(i+1)(i+2)(i+3) \\
= & C_{i} \\
= & f^{+}\left(v_{i}\right)
\end{aligned}
$$

Case(ii): $i$ is even

For $1 \leq i \leq n-1$,

$$
\begin{aligned}
f\left(u_{i}\right)+f\left(u_{i+1}\right)= & \frac{1}{48} i\left(i^{3}+4 i^{2}+2 i-4\right) \\
& +\frac{1}{48} i\left[(i+1)^{3}+5(i+1)^{2}\right. \\
& +7(i+1)+3] \\
= & \frac{1}{48} i\left(i^{3}+4 i^{2}+2 i-4\right) \\
& +\frac{1}{48} i\left(i^{3}+8 i^{2}+20 i+16\right) \\
= & \frac{1}{24} i\left(i^{3}+6 i^{2}+11 i+6\right) \\
= & \frac{1}{24} i(i+1)(i+2)(i+3) \\
= & C_{i} \\
= & f^{+}\left(v_{i}\right)
\end{aligned}
$$

Thus the induced edge labels are the first $n-1$ pentatope numbers.

Hence path $P_{n}$ admits pentatopic sum labeling.

EXAMPLE 8. The pentatopic sum labeling of $P_{9}$ is shown below.

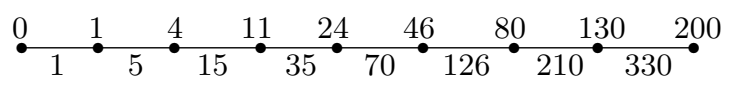

THEOREM 7. The comb $P_{n} \odot K_{1}$ admits pentatopic sum labeling.

Proof. Let $P_{n}: u_{1} u_{2} \ldots u_{n}$ be the path and let $v_{i}=$ $u_{i} u_{i+1}(1 \leq \mathbf{i} \leq \mathbf{n}-1)$ be the edges. Let $w_{1}, w_{2}, \ldots, w_{n}$ be the pendant vertices adjacent to $u_{1}, u_{2}, \ldots, u_{n}$ respectively and $t_{i}=$ $u_{i} w_{i}(1 \leq \mathbf{i} \leq \mathbf{n}-1)$ be the edges.
For $i=1,2, \ldots, n$, define

$$
f\left(u_{i}\right)= \begin{cases}\frac{1}{48}(i-1)\left(i^{3}+5 i^{2}+7 i+3\right) & \text { if } \mathrm{i} \text { is odd } \\ \frac{1}{48} i\left(i^{3}+4 i^{2}+2 i-4\right) & \text { if } \mathrm{i} \text { is even }\end{cases}
$$

and

$$
f\left(w_{i}\right)=\left\{\begin{array}{l}
\frac{1}{48}\left[i^{4}+8 n i^{3}+\left(12 n^{2}+12 n-4\right) i^{2}\right. \\
+\left(8 n^{3}+12 n^{2}-4 n\right) i \\
\left.+\left(2 n^{4}+4 n^{3}-2 n^{2}-4 n+3\right)\right] \quad \text { if } \mathrm{i} \text { is odd } \\
\frac{1}{48}\left[i^{4}+8 n i^{3}+\left(12 n^{2}+12 n-4\right) i^{2}\right. \\
+\left(8 n^{3}+12 n^{2}-4 n\right) i+\left(2 n^{4}\right. \\
\left.\left.+4 n^{3}-2 n^{2}-4 n\right)\right] \quad \text { if } \mathrm{i} \text { is even. }
\end{array}\right.
$$

Then $f\left(u_{i}\right)+f\left(u_{i+1}\right)=f^{+}\left(v_{i}\right)$ for $1 \leq i \leq n-1$

and $f\left(u_{i}\right)+f\left(w_{i}\right)=f^{+}\left(t_{i}\right)$ for $1 \leq i \leq n$.

Thus the induced edge labels are the first $2 n-1$ pentatope numbers.

Hence comb admits pentatopic sum labeling.

EXAMPLE 9. The pentatopic sum labeling of $P_{5} \odot K_{1}$ is shown below.

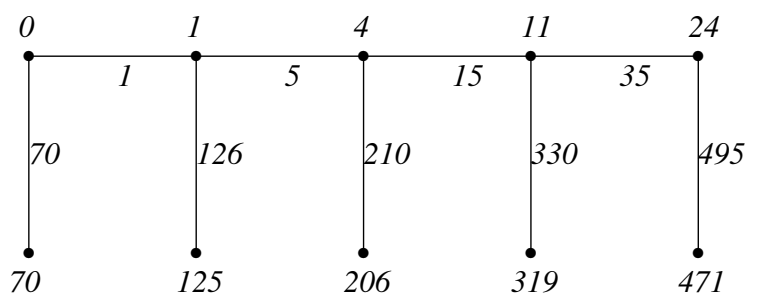

THEOREM 8. The star graph $K_{1, n}$ admits pentatopic sum labeling.

PROOF. Let $v$ be the apex vertex and let $v_{1}, v_{2}, \ldots, v_{n}$ be the pendant vertices of the star $K_{1, n}$. Define

$$
\begin{aligned}
f(v) & =0 \\
\text { and } f\left(v_{i}\right) & =\frac{1}{24} i(i+1)(i+2)(i+3), 1 \leq i \leq n .
\end{aligned}
$$

We see that the induced edge labels are the first $n$ pentatope numbers.

Hence $K_{1, n}$ admits pentatopic sum labeling.

EXAMPLE 10. The pentatopic sum labeling of $K_{1,7}$ is shown below. 


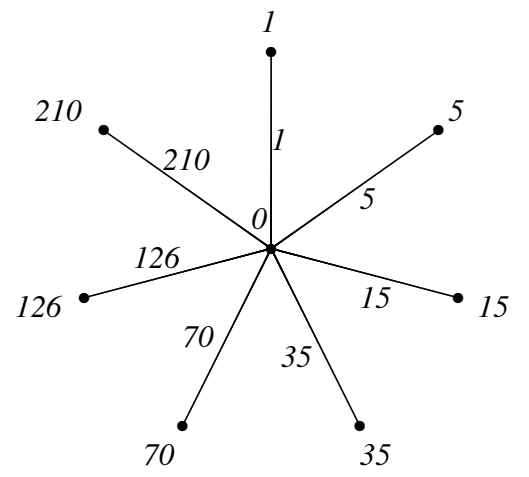

THEOREM 9. $S\left(K_{1, n}\right)$, the subdivision of the star $K_{1, n}$, admits pentatopic sum labeling.

Proof. Let $V\left(S\left(K_{1, n}\right)\right)=\left\{v, v_{i}, u_{i}: 1 \leq i \leq n\right\}$ and $E\left(S\left(K_{1, n}\right)\right)=\left\{v v_{i}, v_{i} u_{i}: 1 \leq i \leq n\right\}$.

Define $f$ by

$$
\begin{aligned}
f(v) & =0, \\
f\left(v_{i}\right) & =\frac{1}{24} i(i+1)(i+2)(i+3), 1 \leq i \leq n
\end{aligned}
$$

and

$$
\begin{aligned}
f\left(u_{i}\right)=\frac{1}{24} & {\left[n^{4}+6 n^{3}+11 n^{2}+6 n\right.} \\
& \left.+2 n i\left(2 n^{2}+3 n i+2 i^{2}+9 n+9 i+11\right)\right], \quad 1 \leq i \leq n .
\end{aligned}
$$

We see that the induced edge labels are the first $2 n$ pentatope numbers.

Hence $S\left(K_{1, n}\right)$ admits pentatopic sum labeling.

EXAMPLE 11. The pentatopic sum labeling of $S\left(K_{1,5}\right)$ is shown below.

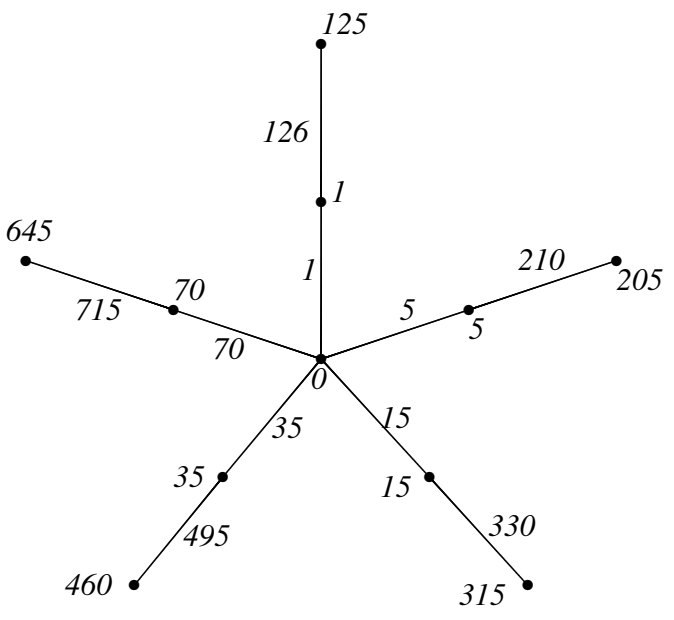

THEOREM 10. The bistar $B_{m, n}$ admits pentatopic sum labeling.
Proof. Let $V\left(B_{m, n}\right)=\left\{u, v, u_{i}, v_{j}: 1 \leq \mathrm{i} \leq \mathrm{m}, 1 \leq \mathrm{j} \leq \mathrm{n}\right\}$ $\underset{E}{\operatorname{and}}\left(B_{m, n}\right)=\left\{u v, u u_{i}, v v_{j}: 1 \leq \mathrm{i} \leq \mathrm{m}, 1 \leq \mathrm{j} \leq \mathrm{n}\right\}$. Define $f$ by

$$
\begin{aligned}
f(u) & =0, \\
f(v) & =1, \\
f\left(u_{i}\right) & =\frac{1}{24}(i+1)(i+2)(i+3)(i+4), 1 \leq i \leq m \\
\text { and } f\left(v_{j}\right)= & \frac{1}{6}(m+j+1)(m+j+2)(m+j+3) \\
& (\times)(m+j+4)-1,1 \leq j \leq n .
\end{aligned}
$$

We see that the induced edge labels are the first $m+n+1$ pentatope numbers.

Hence $B_{m, n}$ admits pentatopic sum labeling.

EXAMPLE 12. The pentatopic sum labeling of $B_{4,3}$ is shown below.

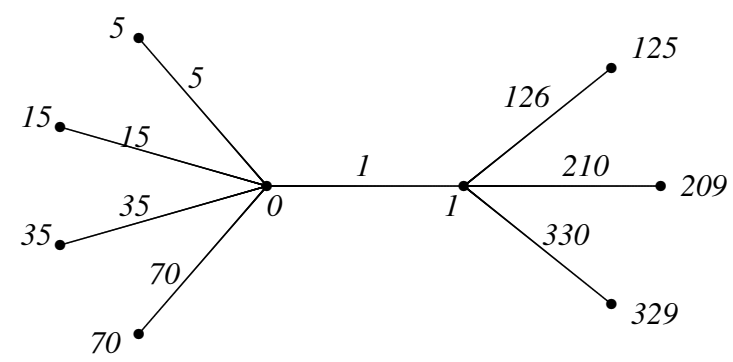

\section{CONCLUDING REMARKS}

As product of three consecutive integers is divisible by 3 ! and product of four consecutive integers is divisible by 4 ! we have introduced tetrahedral sum labeling and pentatopic sum labeling of graphs. These labelings can be extended by using the fact that product of $\mathrm{k}$ consecutive integers is divisible by $\mathrm{k}$ !. Also analogous results for different graphs can be investigated.

\section{ACKNOWLEDGEMENT}

The authors would like to thank Dr.M.Mallikaarjunan, Assistant Professor, Department of Mathematics, C.B.M.College, Coimbatore, India, for helping in the proof reading of this article.

\section{REFERENCES}

[1] F. Harary, Graph Theory, Addition-Wesley, Reading, Mass, 1972.

[2] David M. Burton, Elementary Number Theory, Second Edition,Wm. C. Brown Company Publishers, 1980.

[3] J. A. Gallian, A dynamic survey of graph labeling, Electronic Journal of Combinatorics, 17 (2010), DS6.

[4] S.M.Hegde and P.Shankaran, On triangular sum labeling of graphs, in Labeling of Discrete Structures and Applications, Narosa Publishing House, New Delhi, 2008, 109115 . 